\title{
A graph-theoretic approach to the analysis of international relations
}

\author{
FRANK HARARY and HAROLD MILLER ${ }^{1}$ \\ Research Center for Group Dynamics, University of Michigan
}

In their analysis of international relations, Bernstein and Weldon (1968) present their data in the form of four $35 \times 35$ matrices. They call these

$$
\begin{aligned}
& \text { A }=\text { Reciprocal Defense Relations Matrix } \\
& \mathbf{B}=\text { Organization Matrix } \\
& \mathbf{C}=\text { Trade Matrix } \\
& \mathbf{D}=\text { Embassy Matrix }
\end{aligned}
$$

In the usual manner of using a matrix to represent the adjacency relation in a graph, directed graph, or network (see Harary, Norman, and Cartwright, 1965), the $i$ 'th row and the $i$ 'th column stand for the $i$ 'th nation in their sample of 35 nations (as named in Table 1 ), and the $i, j$ entry of the matrix (in the $i$ 'th row and the $j$ 'th column) tells the relationship of the $i$ 'th nation to the $j$ 'th. Thus, in matrix $\mathrm{A}$ (Bernstein and Weldon, p. 177), the $i, j$ entry, denoted $a(i, j)$, is 1 if nation $i$ has a "reciprocal defense relation" with nation $j, i \neq j$, and

\footnotetext{
${ }^{1}$ The preparation of this article was supported in part by Grant Number MH 10834 from the National Institutes of Health.
}

is 0 otherwise. The diagonal entries are hyphens to indicate irrelevance.

Matrices $C$ and $D$ are also binary (with entries 0 and 1). The $i, j$ entry is 1 in $C$ if nation $j$ is a "principal trader" of nation $i$ and is 1 in $\mathrm{D}$ if nation $i$ has an embassy in nation $j$. The $i, j$ entry of matrix $B$ is the percentage of possible times that nation $i$ is in organizations with nation $j$.

Surprisingly, A is not a symmetric matrix. For example, the United States has a reciprocal defense relation with Ethiopia but Ethiopia does not have one with the United States. There are also other nonreciprocal pairs $(i, j)$ of nations in which $i$ has a "reciprocal defense relation" with $j$. The complete list of these pairs obtained from matrix $A$ (with the corresponding $i, j$ unit entry) is given in Table 2 .

In light of Table 2, we contend that "reciprocal defense relation" is a misnomer and suggest that the name defense commitment relation would be more appropriate. We find it convenient to change $A$ into a symmetric matrix in order to determine international cliques. Two different methods are used and both involve changing the nonsymmetric re- 
TABLE 1

NATIONS IN THE SAMPLE*

\begin{tabular}{lll}
\hline \hline & 13. West Germany & \\
1. United States & 14. Ireland & 25. U. of S. Africa \\
2. Bolivia & 15. Norway & 26. Pakistan \\
3. Ecuador & 16. Sweden & 27. Japan \\
4. Peru & 17. Austria & 28. Burma \\
5. Colombia & 18. Libya & 30. Ahilippines \\
6. Dominican Rep. & 19. Egypt & 31. Thailand \\
7. Honduras & 20. Syria & 32. Australia \\
8. Panama & 21. Iran & 33. Hungary \\
9. Brazil & 22. Jordan & 34. Yugoslavia \\
10. Uruguay & 24. Ghana & 35. USSR \\
11. Denmark & & \\
\hline
\end{tabular}

*From Bernstein and Weldon (1968, p. 161).

TABLE 2

THE NONRECIPROCAL "RECIPROCAL DEFENSE RELATIONSHIPS"

\begin{tabular}{llc}
\hline \hline Nation $i$ (is committed to defend) & \multicolumn{1}{c}{ Nation $j$} & Matrix entry $(i, j)$ \\
\hline United States & Ethiopia & $(1,23)$ \\
United States & Ghana & $(1,24)$ \\
Iran & United Kingdom & $(21,11)$ \\
Ghána & Australia & $(24,32)$ \\
U. of S. Africa & Australia & $(25,32)$ \\
Pakistan & Australia & $(26,32)$ \\
Philippines & Australia & $(27,32)$ \\
Thailand & Australia & $(31,32)$ \\
USSR & Ghana & $(35,24)$ \\
USSR & U. of S. Africa & $(35,25)$ \\
\hline
\end{tabular}

lation given by matrix $\mathrm{A}$ into a symmetric relation.

As an illustration of the methods, Figure 1(a) represents the digraph ${ }^{2}$ for the reciprocal defense relation with respect to the United States, Ghana, Australia, and Ethiopia. That is, a directed line is drawn from the point $\mathbf{v}_{t}$, representing nation $i$ to $v_{j}$ if and only if the entry $a(i, j)$ is 1 .

In terms of its digraph, a relation is symmetric if and only if every pair of points in the relation is joined by a symmetric pair of

\footnotetext{
${ }^{2}$ In every figure, the points are numbered according to Table 1.
}

directed lines, or equivalently, in terms of the adjacency matrix, if and only if $a(i, j)$ and $a(j, i)$ have the same value ( 0 or 1$)$. From Figure 1 (a) we see that, although $v_{1}$ and $v_{32}$ are joined by a symmetric pair, the other pairs of points in the relation are not. To obtain a symmetric relation, one of two operations can be performed: either join every pair of points in the relation by a symmetric pair of directed lines, or remove all directed lines which do not form part of a symmetric pair. The first operation yields the symmetric closure relation and the second the (maximal) symmetric subrelation; these are shown in Figures $1(b)$ and 1(c) respectively for the relation in Figure 


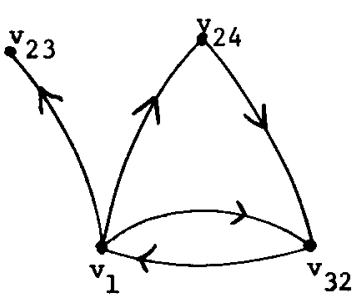

(a)

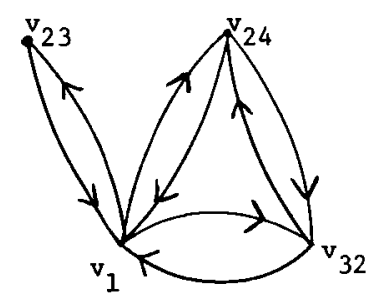

(b)

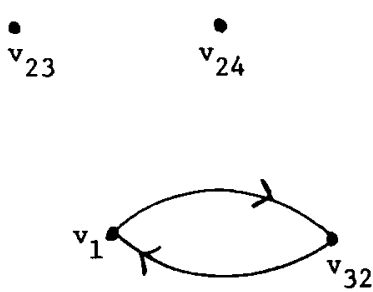

(c)

FIG. 1. An illustration of the symmetric closure relation and symmetric subrelation for the reciprocal defense relations.

1(a). These two operations are equivalent to changing certain entries in the adjacency matrix from 0 to 1 or 1 to 0 , respectively, so that the resultant matrix is symmetric in both cases. Consequently, matrix A was made symmetric by setting the ten $i, j$ unit entries of the matrix cited in Table 2 to zero (for the symmetric subrelation or by setting the corresponding ten $j, i$ zero entries to one (for the symmetric closure relation).

Our object is to analyze matrix $\mathbf{A}$ in terms of the corresponding graph $G$ (compare Harary, 1961, where a similar study was made for the nations involved in the Middle East in 1959) by exploiting the subgraphs of $G$. In the complete graph $\mathrm{K}_{p}$ (see Harary, 1969, for more details concerning the definitions and notation in graph theory used here) with $p$ points, every pair of distinct points are adjacent, that is, they are joined by an undirected line (see Figure 2). A (connected) component of a graph is illustrated by the graph of Figure 3, which has three compo- nents. Note that a component is not necessarily complete. A clique of a graph $\mathrm{G}$ is defined as a maximal complete subgraph, that is, a subgraph which is no longer complete when any other points of $\mathrm{G}$ are included. For example, the graph in Figure 4 has five cliques formed by the following sets of points: $\{a, b, c, d, e\},\{a, f, d\},\{g, d, h\},\{a, i\},\{i, j, k, l\} . \mathrm{A}$ cutpoint of a graph is a point whose removal increases the number of components. For example, the graph in Figure 4 has three cutpoints: $d, a$, and $i$. A bridge is a line whose removal increases the number of components. The graph in Figure 4 has one bridge, namely the line joining points $a$ and $i$.

The graph ${ }^{3}$ representing the symmetric subrelation of the relation of matrix $A$ is shown in Figure 5, and the cliques of the largest component of the graph are listed in Table 3.

${ }^{3}$ For simplicity, the interior lines of the $K_{10}$ clique have been omitted.

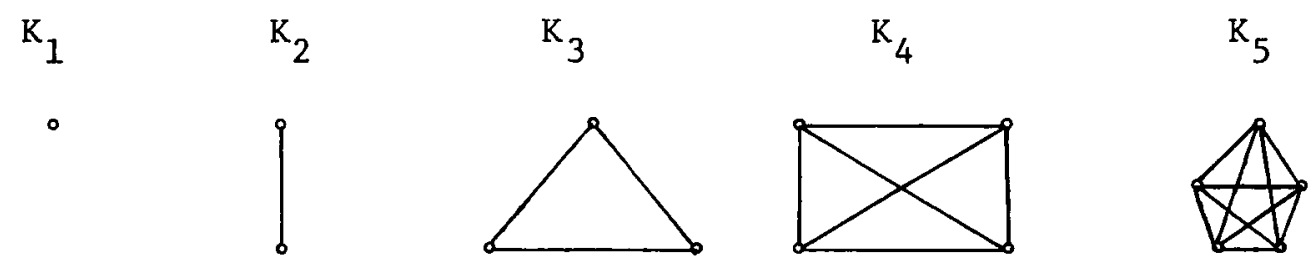

FIG. 2. The smallest complete graphs. 

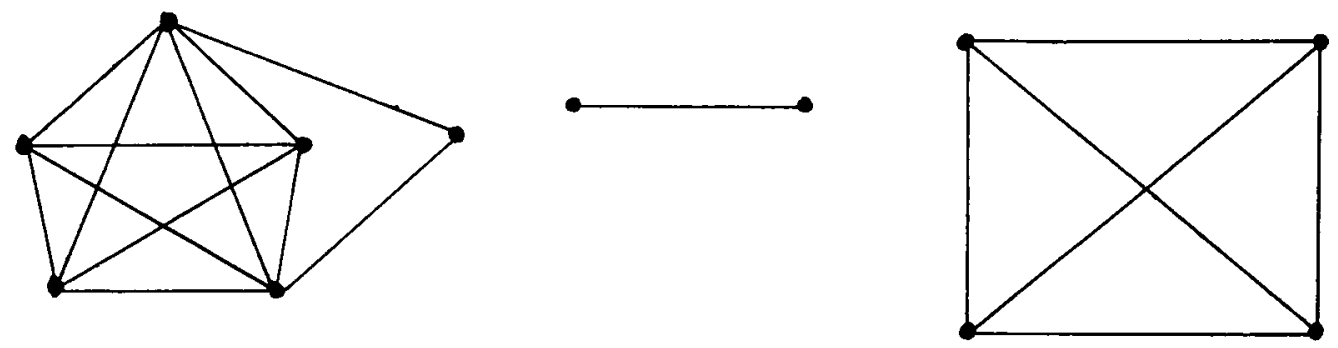

FIG. 3. A graph with three components.

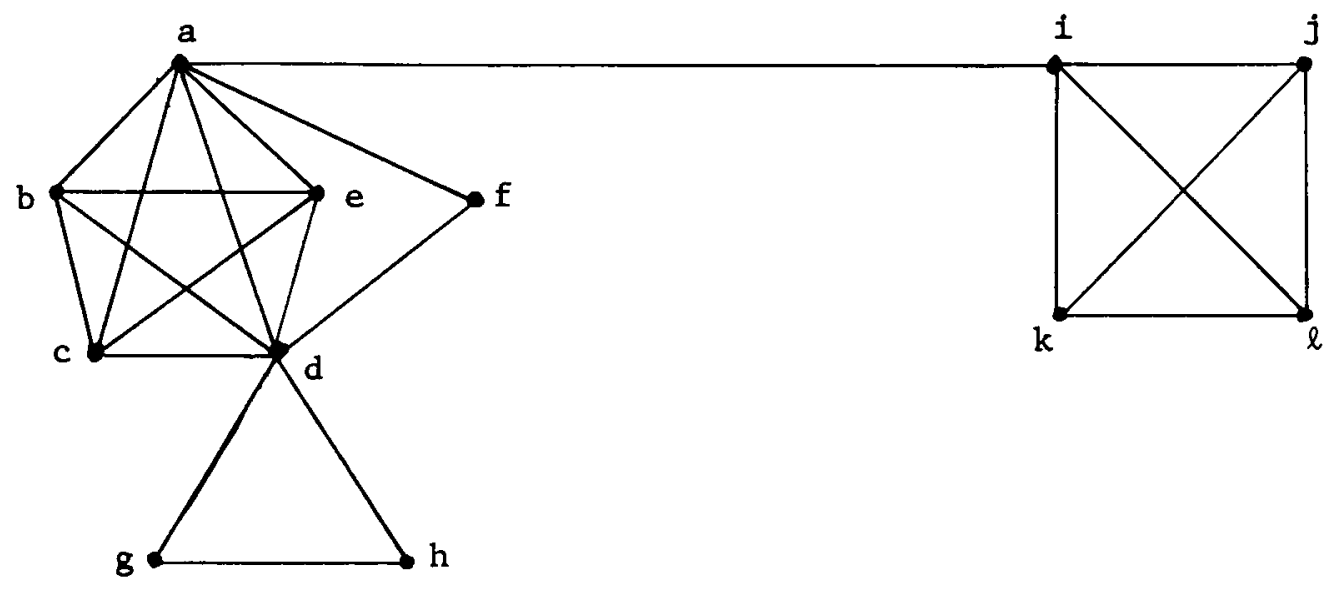

FIG. 4. A graph with five cliques.

Similarly, the graph ${ }^{4}$ for the symmetric closure relation appears in Figure 6 and the cliques in Table 4.

The largest component of the graph of Figure 5 has two cutpoints, the United States and the USSR. It also has two bridges, one between the United States and Japan (a bridge across the Pacific) and the other between the USSR and Hungary. The only nation which is adjacent to both the United States and the USSR is the Union of South Africa! The

"In order to afford a clearer view of the overall structure of the graph, all but two of the interior lines of the two $K_{6}$ cliques have been omitted (the two lines being retained since they also form part of a $K_{1}$ clique) and the interior lines of the $K_{10}$ clique.
TABLE 3

THE CLIQUES OF THE LARGEST COMPONENT OF THE GRAPH OF FIGURE 5

\begin{tabular}{ll}
\hline \hline Description & \multicolumn{1}{c}{ Nations in the clique } \\
\hline $\mathbf{K}_{10}$ & $1,2,3,4,5,6,7,8,9,10$ \\
$\mathbf{K}_{5}$ & $1,11,12,13,15$ \\
$\mathbf{K}_{5}$ & $1,11,26,29,31$ \\
$\mathbf{K}_{4}$ & $1,11,25,26$ \\
$\mathbf{K}_{4}$ & $11,24,25,26$ \\
$\mathbf{K}_{4}$ & $18,19,20,22$ \\
$\mathbf{K}_{3}$ & $1,11,32$ \\
$\mathbf{K}_{3}$ & $1,11,21$ \\
$\mathbf{K}_{3}$ & $24,25,35$ \\
$\mathbf{K}_{2}$ & 1,19 \\
$\mathbf{K}_{2}$ & 1,27 \\
$\mathbf{K}_{2}$ & 11,22 \\
$\mathbf{K}_{2}$ & 33,35 \\
\hline
\end{tabular}




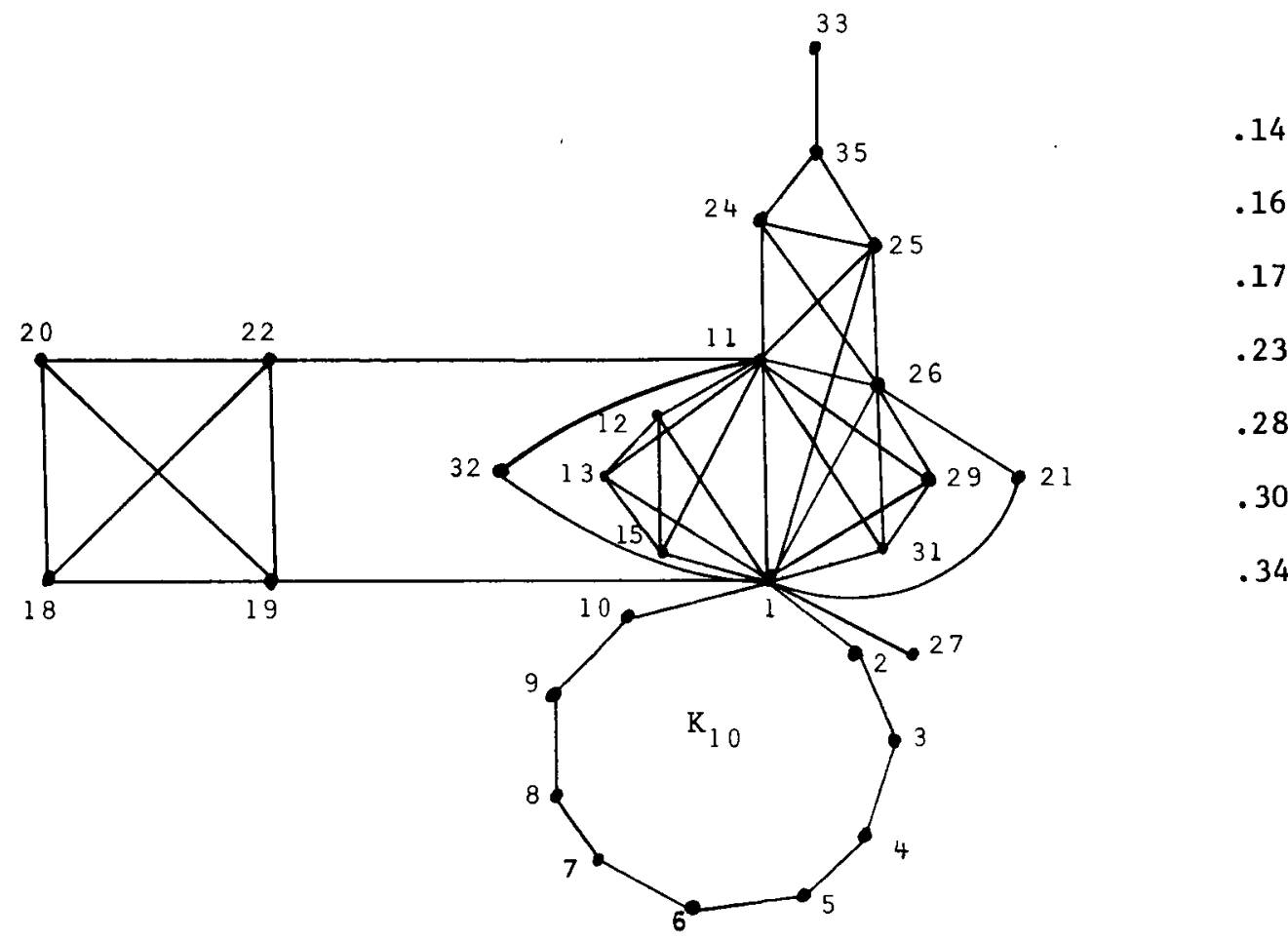

FIG. 5. The graph representing the symmetric subrelation of the reciprocal defense relation.

United States is a member of the largest number of cliques (eight out of 13), followed by

TABLE 4

THE CLIQUES OF THE LARGEST COMPONENT OF THE GRAPH OF FIGURE 6

\begin{tabular}{ll}
\hline \hline Description & Nations in the clique \\
\hline & \\
$\mathbf{K}_{10}$ & $1,2,3,4,5,6,7,8,9,10$ \\
$\mathbf{K}_{6}$ & $1,11,26,29,31,32$ \\
$\mathbf{K}_{6}$ & $1,11,24,25,26,32$ \\
$\mathbf{K}_{5}$ & $1,11,12,13,15$ \\
$\mathbf{K}_{4}$ & $18,19,29,22$ \\
$\mathbf{K}_{4}$ & $1,11,21,26$ \\
$\mathbf{K}_{3}$ & $24,25,35$ \\
$\mathbf{K}_{2}$ & 1,19 \\
$\mathbf{K}_{2}$ & 1,23 \\
$\mathbf{K}_{2}$ & 1,27 \\
$\mathbf{K}_{2}$ & 11,22 \\
$\mathbf{K}_{2}$ & 33,35 \\
\hline
\end{tabular}

the United Kingdom (seven out of 13). These two nations also have the greatest number of relationships ( 21 and 11 , respectively). The largest component of the graph of Figure 6 has the same two cutpoints and bridges as well as a bridge between the United States and Ethiopia. Only Ghana and the Union of South Africa are adjacent to both the United States and the USSR! As in the other graph, the United States is a member of the largest number of cliques (eight out of 12) and has the greatest number of relationships (23) followed by the United Kingdom (five out of 12 cliques and 11 relationships).

Only a few of the many properties of a graph have been described to demonstrate its applicability to the study of international relations. Nevertheless, it should be clear from these illustrative observations that the 


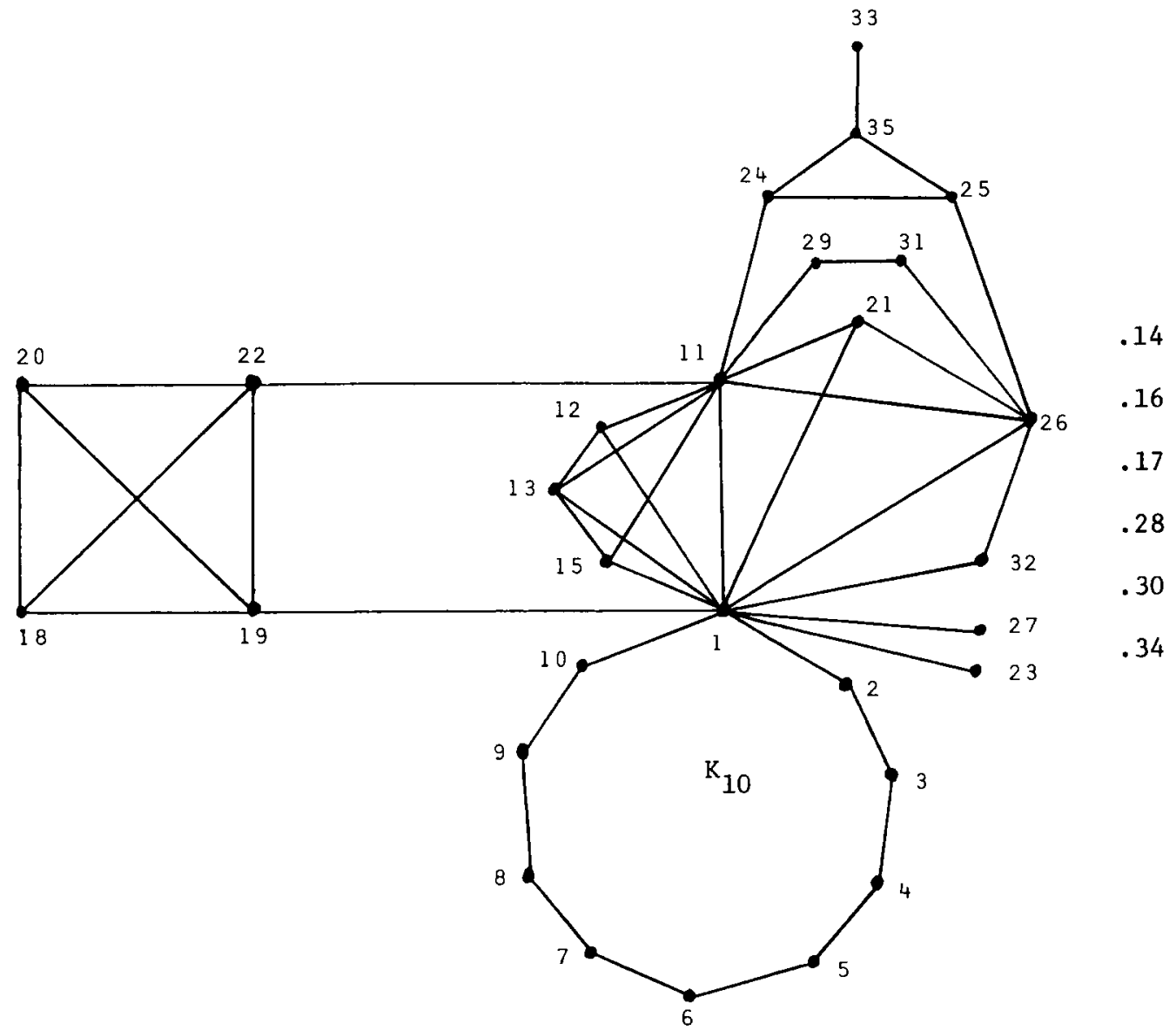

FIG. 6. The graph representing the symmetric closure relation of the reciprocal defense relation.

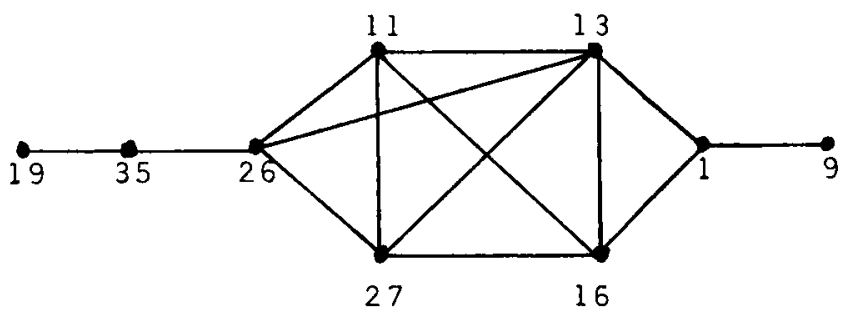

FIG. 7. Graph redrawn from Bernstein and Weldon (1968). 
analytical power provided by a structural approach using concepts from graph theory is far more useful than the mere listing of relationships in matrix form. In fact, Bernstein and Weldon (1968, p. 172) themselves say that "the patterns may be more easily visualized in schematic form," and proceed to construct a "schematic diagram," commonly known as a graph. Nation $i$ and nation $j$ are in the relation (i.e., are adjacent points in the graph) if there is "an overlap in [their] spheres of influence which cannot be attributed to chance more than five times in a hundred." The graph (see Figure 7) has been redrawn from Bernstein and Weldon (1968, p. 173) in order to more clearly show the cliques. The reader will see that there are six cliques formed by the following sets of points: $\{11,13,16,27\},\{11,13,26,27\},\{1,13,16\},\{19,35\}$, $\{26,35\},\{1,9\}$.

\section{REFERENCES}

Bernstein, R. A., and P. D. Weldon. A structural approach to the analysis of international relations. Journal of Conflict Resolution, 1968, 12 (2, June), 159-81.

Harary, F. A structural analysis of the situation in the middle east in 1956, Journal of Conflict Resolution, 1961, 5 (2, June) 167-78.

Wraph Theory. Reading, Mass.: AddisonWesley, 1969.

, R. Norman, and D. Cartwright. Structural Models: An Introduction to the Theory of Directed Graphs. New York: Wiley, 1965. 